\title{
Agnese Silvestri, Il Caso Dreyfus e la Nascita dell'Intellettuale Moderno
}

\section{Maria Emanuela Raffi}

\section{(2) OpenEdition}

1 Journals

\section{Edizione digitale}

URL: http://journals.openedition.org/studifrancesi/4444

DOI: $10.4000 /$ studifrancesi. 4444

ISSN: 2421-5856

\section{Editore}

Rosenberg \& Sellier

\section{Edizione cartacea}

Data di pubblicazione: 1 settembre 2016

Paginazione: 357-358

ISSN: 0039-2944

\section{Notizia bibliografica digitale}

Maria Emanuela Raffi, « Agnese Silvestri, /l Caso Dreyfus e la Nascita dell'Intellettuale Moderno », Studi

Francesi [Online], 179 (LX | II) | 2016, online dal 01 septembre 2016, consultato il 18 septembre 2020. URL : http://journals.openedition.org/studifrancesi/4444; DOI : https://doi.org/10.4000/studifrancesi. 4444

Questo documento è stato generato automaticamente il 18 settembre 2020.

\section{(c)}

Studi Francesi è distribuita con Licenza Creative Commons Attribuzione - Non commerciale - Non opere derivate 4.0 Internazionale. 


\title{
Agnese Silvestri, Il Caso Dreyfus e la Nascita dell'Intellettuale Moderno
}

\author{
Maria Emanuela Raffi
}

\section{NOTIZIA}

AGNESE SILVESTRI, Il Caso Dreyfus e la Nascita dell'Intellettuale Moderno, Milano, FrancoAngeli, 2012, 413 pp.

1 Si segnala, con involontario ritardo, l'ampio e documentato studio di Agnese Silvestri sul caso Dreyfus, sulle circostanze storico-culturali del suo drammatico sviluppo e soprattutto sull'evidente potere della parola e della campagna di stampa che ne accompagnano i diversi momenti. In effetti, in molti momenti della sua attività di scrittore, Zola «rivendica [...] alla scienza e alla letteratura [...] un evidente potere di consolidamento delle istituzioni democratiche», pur utilizzando al tempo stesso e sempre più tale potere, come rileva l'A. attraverso un'ampia documentazione, anche per finalità critiche ed eterodosse nei confronti della classe dirigente. J'Accuse...! sembra segnare sia il punto culminante dell'attacco di Zola all'assetto politico a lui contemporaneo che il limite di tale attacco, dato che lo scrittore è forzato a riconoscere le difficoltà di far passare la sua concezione scientifico-letteraria all'interno della visione politica e popolare. Il problema della distanza fra l'intellettuale e la popolazione rimane per A. Silvestri il problema centrale per chi si accosti al caso Dreyfus, in cui le strategie comunicative dei «dreyfusards» devono fare i conti con il sensazionalismo e le manipolazioni del giornalismo spregiudicato degli avversari.

2 Tutto lo studio che segue queste premesse generali sul problema è costituito dall'esame e dal commento del materiale documentario e forma sedici brevi capitoli nei quali, in ordine cronologico, l'A. prende in esame lettere e articoli che hanno accompagnato le diverse fasi del caso Dreyfus, dalle premesse antisemite degli anni fra il 1886 e il 1892, all'attacco all'“ebreo" del 1894, alla degradazione dell'anno successivo, alla detenzione dal 1895 al 1899, alle prime difese dei «dreyfusards» fra il 1895 e il 1897 e poi via via 
fino al dibattito più acceso degli anni successivi e al J'Accuse...! del 1898. A partire da questa data A. Silvestri comincia a parlare dell'esistenza degli «intellettuali», come «categoria di persone [che] prende coscienza di sé come soggetto sociale collettivo». In questo senso è con la risposta collettiva all'articolo di Zola, pubblicata nell'«Aurore», che nasce questa nuova aggregazione rappresentata da circa mille e cinquecento firme. L'A. ne segue il definirsi attraverso gli articoli che, nel corso del 1898, si interrogano e riflettono sul ruolo di questa nuova figura fino ad arrivare - nel X capitolo - al punto cruciale rappresentato dal "processo Zola», dove lo studio degli articoli è accompagnato dalla documentazione giudiziaria. Gli interventi degli «antidreyfusards», soprattutto di Barrès, occupano il capitolo successivo, mentre gli interventi di Jaurès a dimostrazione della falsità delle accuse mosse a Dreyfus appaiono immediatamente di seguito e preparano il terreno alle riflessioni dell'A. sui commenti alla scelta suicida del colonnello Henry, autore del falso documento accusatore. Articoli, deposizioni e dichiarazioni relativi al processo di Rennes, alla concessione della grazia e alla riabilitazione finale di Dreyfus occupano gli ultimi capitoli di questa intensa e appassionata ricostruzione, attraverso le diverse forme di parola scritta, di uno dei più noti e drammatici "casi" politico-letterari della storia francese. 